\title{
Evaluation of the treatment patterns and economic burden of dysmenorrhea in Japanese women, using a claims database
}

This article was published in the following Dove Press journal:

ClinicoEconomics and Outcomes Research

22 May 2017

Number of times this article has been viewed

\section{Sayako Akiyama' \\ Erika Tanaka ${ }^{2}$ \\ Olivier Cristeau ${ }^{3}$ \\ Yoshie Onishi ${ }^{4}$ \\ Yutaka Osuga ${ }^{5}$}

'Health Economics and Outcomes Research, ${ }^{2}$ Advocacy and External Affairs, Market Access, Bayer

Yakuhin, Ltd., Tokyo, Japan; ${ }^{3}$ Health Economics and Outcome Research, Creativ-Ceutical, Paris, France;

${ }^{4}$ Creativ-Ceutical K.K., Tokyo, Japan; ${ }^{5}$ Obstetrics and Gynecology, Graduate School of Medicine, The University of Tokyo, Tokyo, Japan
Correspondence: Erika Tanaka Advocacy and External Affairs, Market Access, Bayer Yakuhin, Ltd., 6-5 Marunouchi I-chome, Chiyoda-ku, Tokyo 100-8625, Japan

Email erika.tanaka@bayer.com
Purpose: This study aimed to describe treatment patterns and estimate health care resource utilization and associated costs among Japanese women with dysmenorrhea, using a claims database. Methods: This was a retrospective analysis using health insurance data from the Japan Medical Data Center, assessing female patients aged 18-49 years with newly diagnosed primary or secondary dysmenorrhea. Treatment pattern analyses focused on hormonal medications, analgesics, hemostatic agents, traditional Chinese medicine (TCM), and gynecological surgeries. Data were collected on health care resource utilization and costs associated with medications, imaging procedures, and inpatient and outpatient care in both patients and matched controls. Results: The analysis included 6,315 women with dysmenorrhea (3,441 primary; 2,874 secondary). The most commonly prescribed initial therapies were low-dose estrogen progestins (LEPs, 37.7\%) and TCM (30.0\%), with substantial differences between primary (LEPs: $27.4 \%$, TCM: $38.8 \%$ ) and secondary (LEPs: 50.2\%, TCM: 19.5\%) dysmenorrhea cohorts. Surgery was conducted in $<5 \%$ of all patients. Both primary and secondary cohorts of dysmenorrhea had significantly higher mean total health care costs compared to controls within the 1-year period following diagnosis (Case-primary: 191,680 JPY [1,916 USD]; secondary: 246,488 JPY [2,465 USD], Control-primary: 83,615 JPY [836 USD]; secondary: 90,711 JPY [907 USD]) $(p<0.0001)$. After adjusting for baseline characteristics, these costs were 2.2 and 2.9 times higher for primary and secondary dysmenorrhea cohorts, respectively, compared with matched controls, (both $p<0.0001$ ). The main driver of these excess costs was outpatient care, with eight additional physician visits per year among dysmenorrhea patients compared to controls $(p<0.0001)$.

Conclusion: Considerable heterogeneity in treatment patterns was observed, with relatively low utilization of LEPs in patients with primary dysmenorrhea and those treated by internal medicine physicians. Total annual health care costs were approximately $2-3$ times higher in patients with dysmenorrhea compared to women without the condition.

Keywords: dysmenorrhea, women's health, treatment patterns, resource use and costs, economic burden, database analysis

\section{Introduction}

Many Japanese women experience health issues associated with menstruation; these can include menstrual pain also referred to as dysmenorrhea, heavy menstrual bleeding (HMB) also referred to as menorrhagia, and premenstrual syndrome (PMS). ${ }^{1-3}$ Dysmenorrhea is the most common gynecological complaint associated with menstruation with a prevalence of $25 \%$ among all women, and reaching up to $90 \%$ among adolescents. ${ }^{4}$ It has also been reported that one-third of Japanese women require analgesic therapy for dysmenorrhea. ${ }^{5}$ 
In some women, dysmenorrhea cannot be attributed to any specific cause and is referred to as primary or idiopathic. It was reported that $47 \%$ of patients who consulted a physician for menstrual cramping had primary dysmenorrhea, based on a survey in $2000 .{ }^{6}$ In other cases, it is associated with a preexisting gynecological disorder, and the disease is referred to as secondary or organic dysmenorrhea. Preexisting disorders include endometriosis, adenomyosis, and fibroids. Collectively, these are common gynecological complications in women. Regardless of the cause, dysmenorrhea can have a substantial impact on patient quality of life, ${ }^{7,8}$ yet many patients do not seek treatment. ${ }^{9}$ In a patient survey, some untreated women have expressed feelings of resistance or aversion toward seeking therapy, and many suggested that gynecologist consultations were unnecessary for their disorder. ${ }^{1}$ However, a substantial proportion of women who did seek medical treatment agreed that their daily lives were significantly improved after therapy, and it was also estimated that gynecologist visits saved over 7,000 JPY (70 USD) monthly costs per-patient, occurring due to time off work. ${ }^{1}$

According to the guidelines for gynecological practice in Japan, by the Japan Society of Obstetrics and Gynecology (JSOG) and Japan Association of Obstetricians and Gynecologists (JAOG) (2011 edition), low-dose estrogen progestins (LEPs) and nonsteroidal anti-inflammatory drugs (NSAIDs) are primarily recommended for primary dysmenorrhea, and traditional Chinese medicines (TCMs) could be used for primary dysmenorrhea. ${ }^{10}$ Other current clinical practice guidelines for the treatment of dysmenorrhea include the use of over-the-counter analgesics, NSAIDs, and oral contraceptives such as LEPs, progestin-only therapies, and the levonorgestrel-releasing intrauterine system. ${ }^{11,12}$ Two kinds of combined oral contraceptives (COCs) are available on the Japanese market: LEPs, (norethisterone/estrogen and drospirenone/estrogen), which are reimbursed for dysmenorrhea treatment, and the other COCs for contraceptive purposes, which are not reimbursed. Some of these options have demonstrated efficacy in alleviating symptoms among patients with dysmenorrhea. For example, LEPs have been reported to be effective in alleviating symptoms in up to $80 \%$ of women. ${ }^{13}$ Additional studies have shown efficacy of both gonadotropin-releasing hormone $(\mathrm{GnRH})$ analogs and testosterone derivatives for treating underlying causes of secondary dysmenorrhea, such as endometriosis. ${ }^{14-17}$

Existing evidence on treatment patterns, health care resource use, and associated costs in Japanese patients with dysmenorrhea is limited. Information on treatment patterns and the economic burden of disease would be useful to guide the allocation of health care resources for the treatment of dysmenorrhea. Furthermore, the analysis of treatment patterns and resource utilization may shed light on any potential challenges related to the current diagnosis and management of dysmenorrhea in Japan.

The objectives of this study were to describe treatment patterns and estimate health care resource use and costs among Japanese women with newly diagnosed dysmenorrhea in a real-world setting. This included a detailed description of the baseline characteristics and comorbidities of these patients, their initial and subsequent therapies for dysmenorrhea, and the probability of surgical procedure related to dysmenorrhea.

To assess health care resource utilization and costs among patients, a comparison was made between patients with dysmenorrhea (cases) and those without dysmenorrhea (matched control group). Furthermore, all results were differentiated between women with primary and secondary dysmenorrhea and treatment patterns were evaluated by prescriber's specialty. The reason why we took prescriber's specialty into consideration is that the Japanese health care system allows patients free access to any clinic irrespective of specialty.

\section{Methods}

\section{Study design and population}

This was a retrospective claims analysis of the Japan Medical Data Center (JMDC) database, which includes deidentified medical (inpatient and outpatient) and pharmacy claims from up to 3.0 million beneficiaries (both employees and their dependents) from 2005 onward. The JMDC database covers nearly $10 \%$ of the 30 million individuals in the Japanese Social Insurance System, equating to approximately $2.5 \%$ of the total Japanese population. Data were extracted for patients with records between July 1, 2009, and June 30, 2014, and included information on patient demographics, diagnoses, drug prescriptions, medical procedures, medical facility characteristics, and reimbursement costs.

Included patients were women between ages 18 and 49 years who had two separate diagnoses of dysmenorrhea within 3 months. In Japan, patients with dysmenorrhea are generally asked to come back to the clinic within the 3 months following a first medical consultation, as it was considered that only one visit would not be enough for selecting patients requiring medical care for dysmenorrhea. Diagnoses were identified by ICD-10 (International Classification of Diseases 10th revision) codes N944 (primary dysmenorrhea) or N946 (dysmenorrhea, unspecified) or one of the following Medical Information System Development Center (MEDIS-DC) 
standard disease names: functional dysmenorrhea, menstrual pain, or dysmenorrhea. MEDIS-DC consists of standardized names of diseases and injuries for reimbursement by the National Health Insurance. ${ }^{18}$ The index date was defined as the date of the first diagnosis of dysmenorrhea. Primary dysmenorrhea was defined as patients without underlying causes (endometriosis, adenomyosis, and fibroids) during the preindex period and from 60 days after the index date, whereas secondary dysmenorrhea was defined as patients presenting at least one diagnosis of one of these conditions during this period. This criterion was defined based on the publications by Copher et $\mathrm{al}^{19,20}$ and an assumption that imaging procedures for detecting underlying causes would be performed within 2 months following the first visit. Patients were also required to have insurance records for at least 6 months with a diagnosis of dysmenorrhea prior to their initial diagnosis (preindex period) and for a continuous postindex period of at least 1 year, to be included in the study. Patients were excluded if they had been diagnosed with gynecological cancers or bleeding disorders during the study period (including both pre- and postindex), if they underwent gynecological surgery (hysterectomy, endometrial ablation, or myomectomy) during the preindex period, or if they were treated with hormonal agents during the preindex period (Figure 1).

For the resource utilization analysis, matched controls were identified among females in the JMDC database who did not suffer from dysmenorrhea (ie, no diagnosis between July 1, 2009, and June 30, 2014). Controls and cases were matched 2:1 by year of birth (within 5 years), Charlson Comorbidity Index (CCI) at baseline $( \pm 15$ days from index date), and at least the same 18 months of follow-up period.

\section{Treatment patterns}

The study assessed therapies that fit into one of the following categories: hormonal treatments (LEPs, progestin, testosterone derivatives, and GnRH analogs), NSAIDs, hemostatic agents, and TCM therapies. Relevant gynecological surgeries, including hysterectomies, endometrial ablations, and myomectomies, were also considered.

The treatment patterns were analyzed based on prescription records, with a treatment line defined as a group of consecutive prescriptions of the same agent or combination of agents (combination therapy), with no interruption exceeding 90 days. For each treatment line, the start date, end date, duration, and treatment category were determined. When a period exceeding 90 days was observed between the end of one treatment line and the beginning of a new one, a treatment discontinuation was deemed to have occurred.
Therapy switches were defined when an overlap shorter than 21 days between the end of one therapy and the beginning of a new one was observed and the interval did not exceed 90 days. If an overlap occurred for 21 days or more, however, this was considered a combination therapy. Because surgery was considered as a permanent treatment, a separate combination therapy type (surgery combination) was assigned. The drug categories assessed in this study are described in Table 1.

Treatment patterns were analyzed among the entire cohort and according to the type of dysmenorrhea (primary or secondary). A subgroup analysis was also conducted according to the prescriber's specialty for the first treatment line.

\section{Resource utilization and cost calculations}

The resources used and their associated costs were calculated based on the 1-year follow-up period after the index date for each patient. Data were collected on inpatient costs, outpatient costs, and costs associated with both prescriptions and imaging procedures. From this information, total health care costs were calculated.

Resource utilization data collected in this analysis included the number of inpatient admissions, cumulative length of stay, number of outpatient visits, and number of imaging procedures. Imaging procedures, which included echography, computed tomography scan, and magnetic resonance imaging, were determined to be related to a diagnosis of dysmenorrhea when they occurred within a 1-month period around the date of the diagnosis ( \pm 15 days). The use of imaging procedures in controls was evaluated during the same 1-month period as it was for their corresponding matched cases.

\section{Statistical analysis}

Nonadjusted comparisons were conducted for outcomes with respect to primary vs secondary dysmenorrhea and cases vs controls. For continuous variables, a Fisher's $F$-test was conducted to test the assumption of homoscedasticity. Mean values were then compared using a Student's $t$-test (for variables showing homoscedasticity) or Satterthwaite test (heteroscedasticity). For categorical variables, a $\chi^{2}$ or Fisher's exact test (in cases of insufficient theoretical size of subgroups) was performed to compare the distributions.

Time-to-event variables were assessed using Kaplan-Meier curves and log-rank tests for between-group comparisons. Time to "competing events" (ie, end-of-treatment events such as treatment switch, discontinuation of treatment, surgery, addon therapy, and step-down therapy) were analyzed using the competing risks survival method, estimating the cumulative 


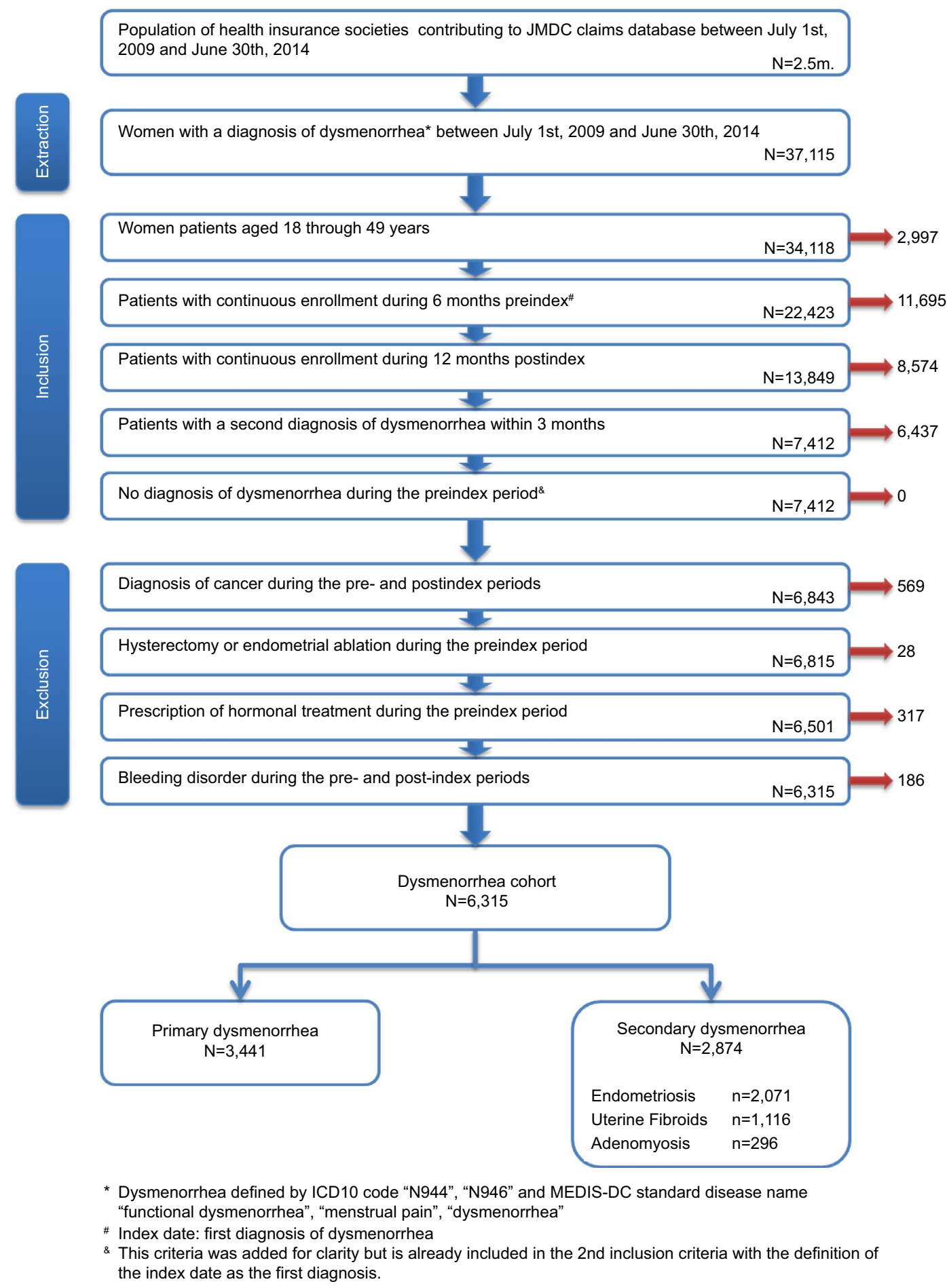

Figure I Flow chart of selection process for patients with dysmenorrhea.

Abbreviations: JMDC, Japan Medical Data Center; ICDI0, International Classification of Disease I0th revision; MEDIS-DC, Medical Information System-Development Center.

incidence. Differences between groups were assessed using a Gray's test.

The analyses of costs and resources were conducted through Generalized Linear Models (GLMs), testing normal, Poisson, negative binomial, zero-inflated Poisson, and zeroinflated negative binomial distributions. The identity and log link functions were investigated. The goodness of fit of the models was assessed with the deviance, and the dispersion with the Pearson $\chi^{2}$ value divided by the number of degrees of freedom. The models minimizing the deviance or with the best compromise deviance/dispersion (in case of high dispersion) were retained. Models were adjusted for age, the category (based on quartiles) of total health care costs over the preindex period, the quantity corresponding to the 
Table I Type of treatment evaluated in the analysis

\begin{tabular}{|c|c|c|c|c|}
\hline Hormonal treatments & Analgesic agents & Hemostatic agents & TCM therapies & Surgical procedures \\
\hline $\begin{array}{l}\text { LEP: } \\
\text { - Low-dose } \\
\text { norethisterone } \\
\text { and estrogen } \\
\text { - Low-dose drospirenone } \\
\text { and estrogen } \\
\text { Progestin: } \\
\text { - Dienogest } \\
\text { Testosterone derivatives: } \\
\text { - Danazol } \\
\text { GnRH analog: } \\
\text { - Buserelin } \\
\text { - Leuprorelin } \\
\text { - Goserelin }\end{array}$ & $\begin{array}{l}\text { NSAIDs, prescribed with } \\
\text { a diagnosis of pain*: } \\
\text { - Indometacin } \\
\text { - Sulindac } \\
\text { - Diclofenac } \\
\text { - Etodolac } \\
\text { - Acemetacin } \\
\text { - Proglumetacin } \\
\text { - Piroxicam } \\
\text { - Tenoxicam } \\
\text { - Lornoxicam } \\
\text { - Meloxicam } \\
\text { - Ibuprofen } \\
\text { - Naproxen } \\
\text { - Ketoprofen } \\
\text { - Fenoprofen } \\
\text { - Fengufen } \\
\text { - Flurbiprofen } \\
\text { - Tiaprofenic acid } \\
\text { - Oxaprozin } \\
\text { - Alminoprofen } \\
\text { - Mefenamic acid } \\
\text { - Tolfenamic acid } \\
\text { - Flufenamic acid } \\
\text { - Celecoxib } \\
\text { - Nabumetone } \\
\text { - Acetylsalicylic acid }\end{array}$ & 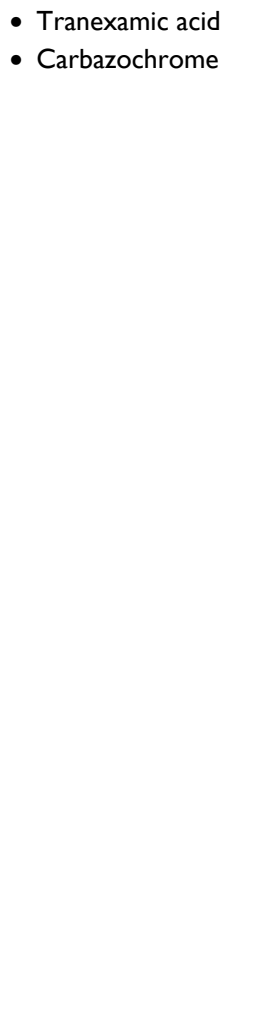 & $\begin{array}{l}\text { - Tokishakuyakusan extract } \\
\text { - Kamishoyosan extract } \\
\text { - Keishibukuryogan extract } \\
\text { - Tokishakuyakusankabushi } \\
\text { extract } \\
\text { - Daiobotampito extract } \\
\text { - Unseiin extract } \\
\text { - Tokakujokito extract } \\
\text { - Unkeito extract } \\
\text { - Goshakusan extract } \\
\text { - Tokikenchuto extract } \\
\text { - Kyukichoketsuin extract }\end{array}$ & $\begin{array}{l}\text { Complete ablation: } \\
\text { - Hysterectomy } \\
\text { Partial ablation: } \\
\text { - Endometrial ablation } \\
\text { - Myomectomy } \\
\text { - Uterine-artery } \\
\text { embolization }\end{array}$ \\
\hline
\end{tabular}

Notes: *As suggested by experts, physicians may prescribe NSAIDs to patients, associated with a diagnosis of headache, acute pain, pain in the abdomen (upper, lower, or unspecified), low back pain, and pelvic and perineal pain. "Surgical procedure codes were identified based on the medical procedure index available on the MHLW website. ${ }^{23}$ Abbreviations: GnRH, gonadotropin-releasing hormone; LEP, low-dose estrogen progestin; NSAIDs, nonsteroidal anti-inflammatory drugs; TCM, traditional Chinese medicine.

modeled variable computed over the preindex period (eg, the inpatient costs over the preindex period while modeling the inpatient costs over the 1-year postindex period), hospitalization during the preindex period, and the type of health insurance membership (employee or dependent). All analyses were performed using SAS Analytics Pro release 9.3 and $\mathrm{R}$ (version 3.2.1, SAS Institute, Cary, NC, USA) software (for time-to-event data). In general, a $p$-value of $\leq 0.05$ was determined to represent statistical significance.

This study was approved by the Ethics Committee of the nonprofit organization, Clinical Research Promotion Network Japan. The information available in the JMDC database is anonymized. The informed consent of patients was not applicable based on the Ethical Guidelines for Epidemiological Research issued by the Ministry of Health, Welfare and Labor.

\section{Results}

\section{Patient characteristics}

A total of 6,315 patients with dysmenorrhea, 3,441 (54.5\%) primary and 2,874 (45.5\%) secondary, were identified, with an average follow-up duration of $4.0 \pm 0.9$ years. Patients with secondary dysmenorrhea were significantly older compared with patients with primary dysmenorrhea (35.7 \pm 8.0

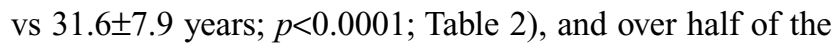
secondary dysmenorrhea cohort were older than 35 years (59.3\% vs $36.8 \%$ in the primary dysmenorrhea cohort). Primary ovarian dysfunction was the most common comorbidity $(22.1 \%)$ at baseline and was more frequent in patients with primary dysmenorrhea than secondary dysmenorrhea $(25.0 \%$ vs $18.6 \% ; p<0.0001$; Table 2$)$. The other comorbidities were more frequently reported in patients with secondary dysmenorrhea than primary dysmenorrhea, including erosion and ectropion of cervix uteri ( $21.8 \%$ vs $15.6 \% ; p<0.0001)$, anemia (19.7\% vs $6.2 \% ; p<0.0001)$, acute vaginitis ( $14.5 \%$ vs $10.2 \%$; $p<0.0001)$, and low back pain $(11.2 \%$ vs $8.1 \%$; $p<0.0001)$, respectively. Over three-quarters of patients had a CCI score of 0 , and less than $4 \%$ had a score greater than 1 (Table 2 ).

For both cohorts, first diagnoses of dysmenorrhea were reported in clinics (0-19-bed) $(76.4 \%)$ and in hospitals $(\geq 20$ bed) $(23.6 \%)$. In more detail, $81.3 \%$ of primary and $70.6 \%$ of secondary dysmenorrhea cases were reported in clinics and $18.7 \%$ of primary and $29.4 \%$ of secondary dysmenorrhea 
Table 2 Patient baseline characteristics at index date

\begin{tabular}{|c|c|c|c|c|}
\hline Characteristics & Primary $(\mathrm{N}=3,44 \mathrm{I})$ & Secondary $(N=2,874)$ & Total $(\mathbf{N}=6,3 \mid 5)$ & $p$-value \\
\hline Mean (SD) age, years, at index date & $31.64(7.92)$ & $35.65(8.01)$ & $33.47(8.21)$ & $<0.0001$ \\
\hline \multicolumn{5}{|l|}{ Age, years, category, N (\%) } \\
\hline $18-34$ & $2,174(63.18)$ & I, I $70(40.7 I)$ & $3,344(52.95)$ & \multirow[t]{3}{*}{$<0.000$ I } \\
\hline $35-39$ & $608(17.67)$ & $609(21.19)$ & $1,217(19.27)$ & \\
\hline $40-49$ & $659(19.15)$ & $1,095(38.10)$ & I,754 (27.78) & \\
\hline \multicolumn{5}{|l|}{ Type of member, $\mathrm{N}(\%)$} \\
\hline Individual & $\mathrm{I}, 570(45.63)$ & I,289 (44.85) & $2,859(45.27)$ & \multirow[t]{2}{*}{0.54} \\
\hline Family & I,87I (54.37) & I,585 (55.15) & $3,456(54.73)$ & \\
\hline \multicolumn{5}{|l|}{ Disease status, N (\%) } \\
\hline Dysmenorrhea only & $3,385(98.37)$ & $2,734(95.13)$ & $6,119(96.90)$ & \multirow[t]{2}{*}{$<0.000$ I } \\
\hline Both dysmenorrhea and $\mathrm{HMB}$ & $56(1.63)$ & $140(4.87)$ & $196(3.10)$ & \\
\hline \multicolumn{5}{|l|}{ Underlying conditions, N (\%) } \\
\hline Endometriosis & 0 & $2,071(72.06)$ & $2,071(72.06)$ & \multirow[t]{3}{*}{ NA } \\
\hline Adenomyosis & 0 & $296(10.30)$ & $296(10.30)$ & \\
\hline Fibroids & 0 & I, $166(40.57)$ & $\mathrm{I}, 166(40.57)$ & \\
\hline \multicolumn{5}{|l|}{$\mathrm{CCl}$ category, $\mathrm{N}(\%)$} \\
\hline 0 & $2,706(78.64)$ & $2,269(78.95)$ & $4,975(78.78)$ & \multirow[t]{5}{*}{0.61} \\
\hline 1 & $614(17.84)$ & $494(17.19)$ & $\mathrm{I}, 108(17.55)$ & \\
\hline 2 & $104(3.02)$ & $95(3.31)$ & $199(3.15)$ & \\
\hline 3 & $15(0.44)$ & II (0.38) & $26(0.4 I)$ & \\
\hline $4+$ & $2(0.06)$ & $5(0.17)$ & $7(0.11)$ & \\
\hline \multicolumn{5}{|l|}{ Top five comorbidities at baseline, $\mathrm{N}(\%)$} \\
\hline $\begin{array}{l}\text { Primary ovarian dysfunction (ovarian } \\
\text { insufficiency, luteal phase deficiency) }\end{array}$ & $861(25.02)$ & $533(18.55)$ & I,394 (22.07) & $<0.0001$ \\
\hline Erosion and ectropion of cervix uteri & $538(15.63)$ & $625(21.75)$ & I,I63 (I8.42) & $<0.0001$ \\
\hline Iron deficiency anemia, unspecified & $214(6.22)$ & $566(19.69)$ & $780(12.35)$ & $<0.0001$ \\
\hline Acute vaginitis & $351(10.20)$ & $417(14.5 I)$ & $768(12.16)$ & $<0.0001$ \\
\hline Low back pain & $280(8.14)$ & $322(11.20)$ & $602(9.53)$ & $<0.0001$ \\
\hline
\end{tabular}

Abbreviations: $\mathrm{CCl}$, Charlson Comorbidity Index; HMB, heavy menstrual bleeding; SD, standard deviation.

cases were reported in hospitals. Patients who were diagnosed in large hospitals ( $\geq 500$-bed) represented $5.7 \%$ of the primary dysmenorrhea cohort and $11.7 \%$ of the secondary dysmenorrhea cohort. Thus, patients in the secondary dysmenorrhea cohort were significantly more likely to be diagnosed in hospitals $(p<0.0001)$ and very large $(\geq 500$-bed $)$ facilities $(p<0.0001)$ compared to the primary dysmenorrhea cohort. The majority of patients were diagnosed in obstetrics and gynecology facilities (53.0\% and $64.6 \%$ for primary and secondary dysmenorrhea cohorts, respectively), whereas the remainder were typically diagnosed in general internal medicine facilities $(32.1 \%$ and $27.6 \%$ for primary and secondary dysmenorrhea cohorts, respectively). At least one diagnostic imaging procedure within 2 weeks before or after diagnosis was reported for $38.7 \%$ of patients with primary dysmenorrhea and $69.2 \%$ of those with secondary dysmenorrhea; in most cases, this was echography. Among women with secondary dysmenorrhea, $4.9 \%$ also presented with a diagnosis of heavy menstrual bleeding at baseline.

\section{Treatment patterns}

A total of $83.4 \%$ of patients in the primary dysmenorrhea cohort and $89.2 \%$ in the secondary dysmenorrhea cohort were treated with at least one pharmacological agent or surgical procedure. Median times to any treatment initiation after diagnosis of dysmenorrhea were 9 and 3 days, in primary and secondary dysmenorrhea cohorts, respectively. In the primary dysmenorrhea cohort, TCM therapies were the most frequently prescribed first-line treatment and were administered in $38.8 \%$ of all patients with primary dysmenorrhea (including treated and untreated patients), LEPs were prescribed in $27.4 \%$ of patients, and the use of hemostatic agents was reported in $12.8 \%$ (Figure 2). In the secondary dysmenorrhea cohort, the proportion of patients treated with LEPs was higher, with $50.2 \%$ receiving these agents as a first-line treatment, whereas $19.5 \%$ were treated with TCM therapies (Figure 3). Substantial differences in the treatment of dysmenorrhea were observed according to the specialty of the prescriber: LEPs were prescribed in first line by obstetricians and gynecologists in $57.2 \%$ and $67.7 \%$ of treated patients from the primary and secondary dysmenorrhea cohorts, respectively, while internal medicine physicians prescribed LEPs to $11.6 \%$ and $35.7 \%$ of treated patients, respectively.

Hemostatic agents were prescribed in first line in 3.8\% and $3.4 \%$ by obstetricians and gynecologists, and in $25.4 \%$ 
and $21.8 \%$ by internal medicine physicians, of treated patients in the primary and secondary dysmenorrhea cohorts, respectively. TCMs were prescribed in first line in 34.0\% and 20.6\% by obstetricians and gynecologists, and in $56.1 \%$ and $25.3 \%$ by internal medicine physicians, of treated patients in the primary and secondary dysmenorrhea cohorts, respectively.

The time to treatment discontinuation was longer for LEPs compared with other therapies. In patients with primary dysmenorrhea, 35.1\% were still receiving LEP treatment at 1 year, compared to $10.4 \%$ for TCM therapies. Similarly, $33.9 \%$ of patients with secondary dysmenorrhea continued to receive LEPs at 1 year, while only $11.5 \%$ continued taking TCM therapies for this duration. Hemostatic agents and NSAIDs were administered only briefly, with a median treatment duration of 5 days for both agents, regardless of the cohort.

Of those patients who did not continue with their first-line treatment for the first 12 months postindex date, only a minority switched to a new therapy $(17.1 \%$ and $21.3 \%$ for the primary and secondary dysmenorrhea cohorts, respectively) and most did not receive any second-line treatment $(73.7 \%$ and $63.3 \%$, respectively). The most widely used treatments in both second and third lines were also LEPs and TCM therapies. The probability of surgery at 1 year was $4 \%$ in the secondary dysmenorrhea cohort and $0.2 \%$ in the primary dysmenorrhea cohort.

\section{Resource utilization and costs associated with dysmenorrhea}

Patients with dysmenorrhea and the matched control cohort of patients without dysmenorrhea had similar baseline characteristics and resource utilization levels prior to the index period, with all groups reporting a mean number of inpatient admissions of 1.1 for the pre-index period. The analysis of inpatient care demonstrated a significant increase in the number of hospital admissions and length of stay due to dysmenorrhea in the secondary dysmenorrhea cohort vs the controls (5.7 additional admissions per 100 persons over 12 months; 2.2 times longer cumulative length of stay; both $p<0.0001$ ), whereas there was no change in admissions compared to controls in the primary dysmenorrhea cohort. Outpatient care visits occurred substantially more often in patients with dysmenorrhea vs controls, with primary and secondary dysmenorrhea cohorts reporting 8.0 and 8.5 additional outpatient visits over 12 months, respectively, after adjusting for baseline characteristics (both $p<0.0001$; Table 3).

Primary and secondary dysmenorrhea cohorts had significantly higher mean total health care costs than controls (mean \pm SD: 191,680 JPY (1,917 USD) \pm 261,226 JPY (2,612 USD) vs 83,615 JPY (836 USD) $\pm 246,093$
JPY (2,461USD) for primary dysmenorrhea cohort and 246,488 JPY (2,465 USD) $\pm 295,936$ JPY (2,959 USD) vs 90,711 JPY (907 USD) \pm 297,513 JPY (2,975 USD) for secondary dysmenorrhea cohort; $p<0.001$; Table 3 ). After adjusting for baseline characteristics, costs were 2.2 times and 2.9 times higher in patients in the primary and secondary dysmenorrhea cohorts, respectively, than controls (both $p<0.0001$ ). Furthermore, total costs in secondary cases were higher by $33.5 \%$ compared to primary cases $(p<0.001$; Table 3$)$.

\section{Discussion}

This analysis demonstrated that the most commonly prescribed treatments for dysmenorrhea in Japan - excluding non-reimbursed agents - were LEPs and TCM therapies, and patients with secondary dysmenorrhea had the highest utilization rates of LEPs. Important differences in treatment patterns were observed according to the specialty of the prescriber of the first treatment line: obstetricians and gynecologists mainly prescribed LEPs, whereas internal medicine physicians prescribed TCM therapies most frequently. This might be due to differences in severity of dysmenorrhea among women who seek medical care from obstetricians and gynecologists vs internal medicine physicians and because women suffering from more severe dysmenorrhea may be more likely to consult an obstetrician or gynecologist and to receive LEPs. Our findings were generally compatible with the guidelines for gynecological practice by the JSOG and JAOG 2011 edition. ${ }^{10}$ Although such guidance suggests NSAID treatment for initial pain relief, our findings show relatively low levels of NSAID use. One of the reasons may be that many patients may have used over-the-counter therapies including NSAIDs, which are not captured by the database. ${ }^{1}$ Persistence on LEPs was higher in this analysis than for any of the other therapies assessed, including TCM therapies. It was found by adding up the proportions of patients without defined treatment $(13.0 \%$ of both primary and secondary dysmenorrhea cases) and those with hemostatic agents $(11.2 \%)$ and NSAIDs $(1.6 \%)$ that around $25 \%$ of patients diagnosed with dysmenorrhea received no reimbursed treatment or short-term treatments only. Surgery was rarely used to treat dysmenorrhea.

This study also demonstrated that affected patients have more frequent physician visits, equating to roughly one additional visit every 45 days that can be attributed to dysmenorrhea. This high frequency suggests that the disease has a strong impact on the quality of life of these patients. Furthermore, dysmenorrhea does require inpatient care in some cases, with approximately six additional inpatient 


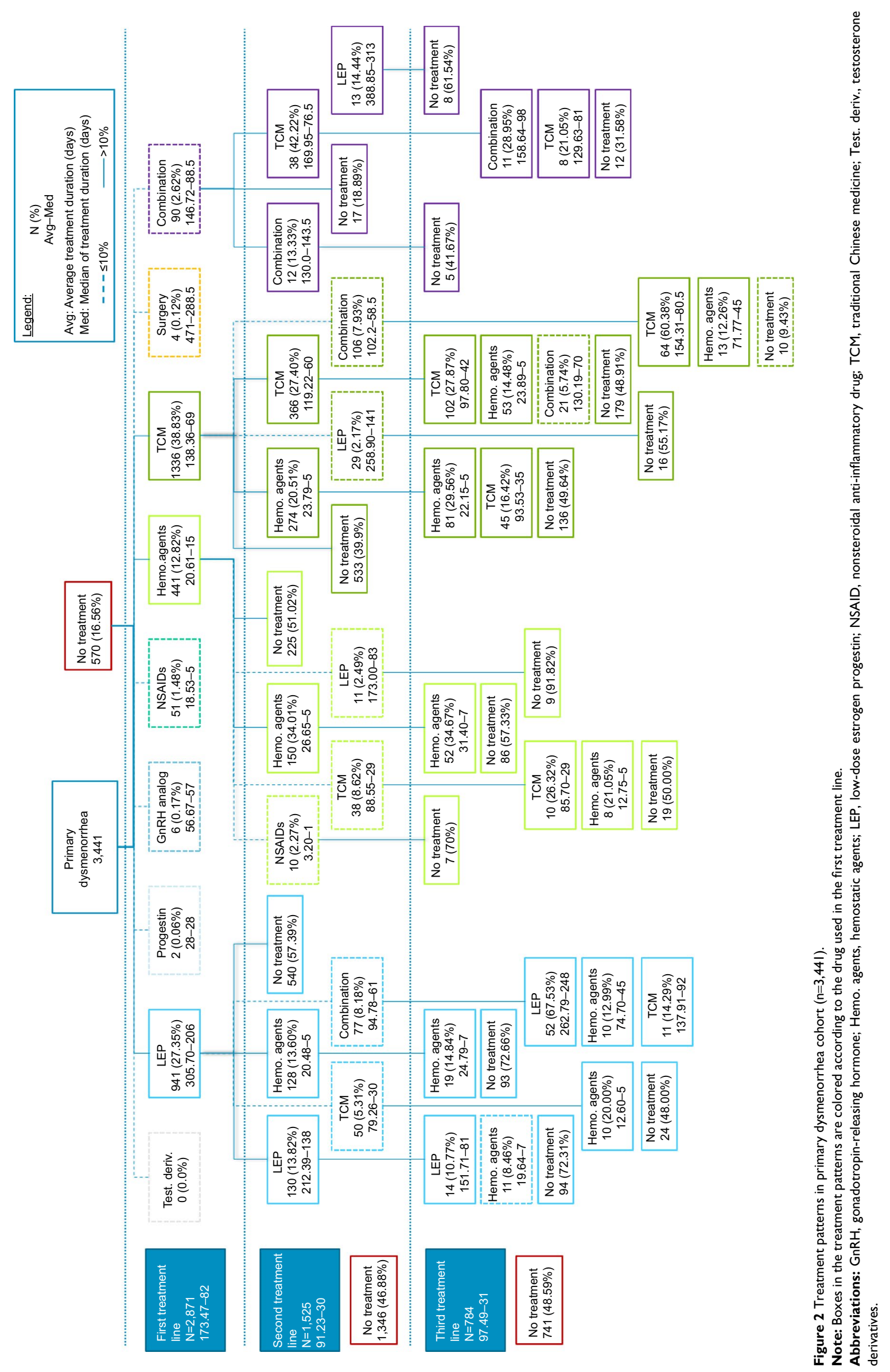




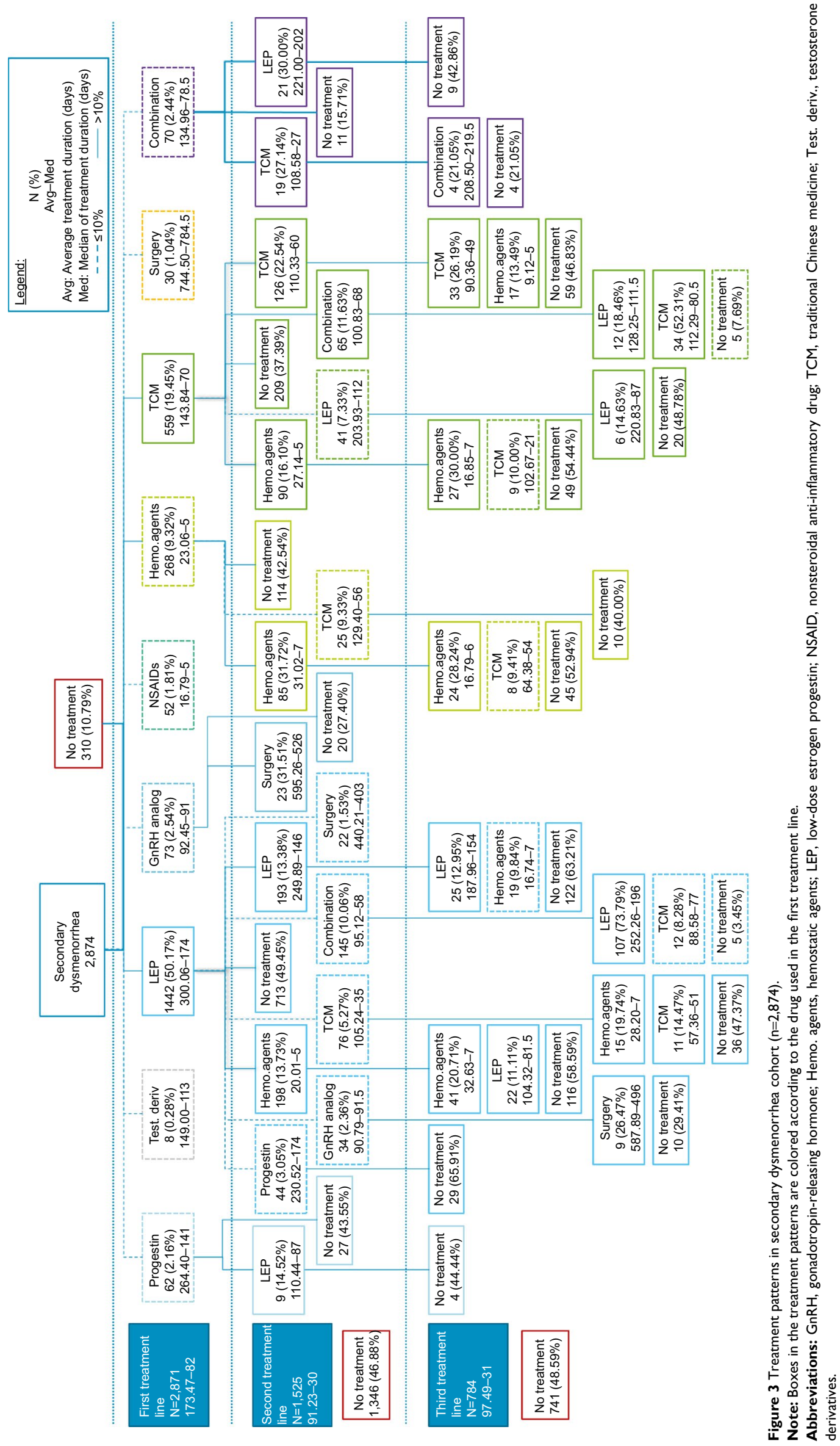




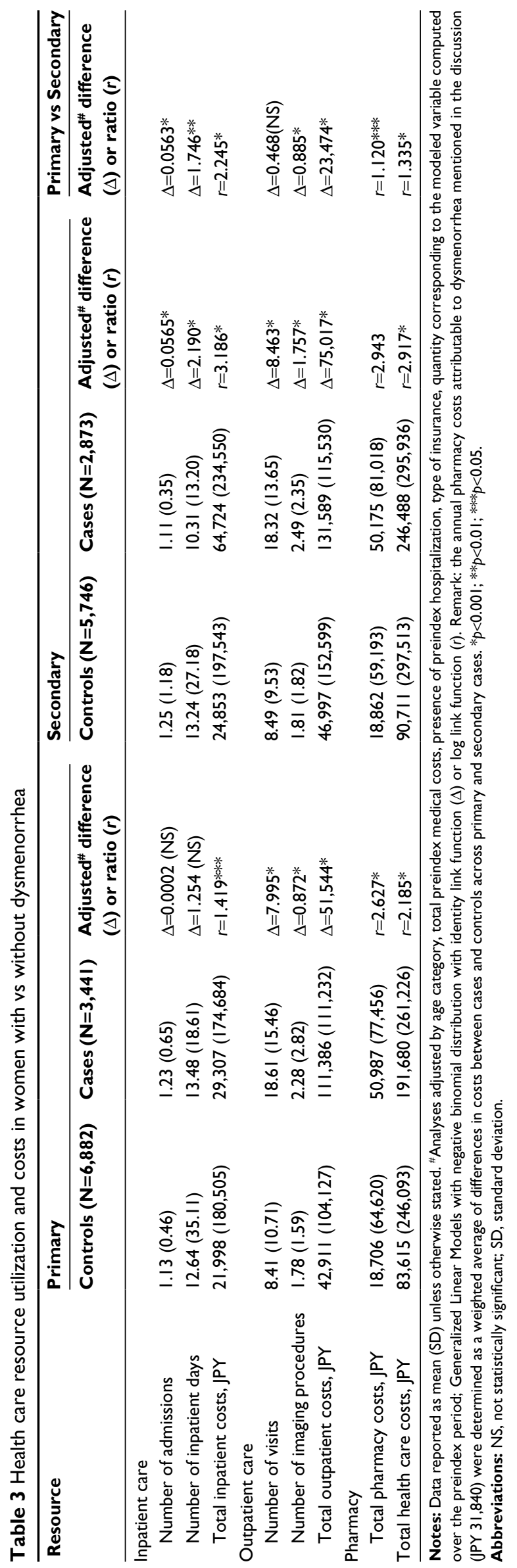

admissions per 100 patients over 12 months attributed to dysmenorrhea in patients with secondary dysmenorrhea. As a result, health care costs in women with dysmenorrhea are two to three times higher than costs in women who do not suffer from this disorder.

Estimates of the prevalence of dysmenorrhea in Japan ranged from $15.8 \%$ to $25 \%{ }^{4,21} \mathrm{~A}$ survey conducted in 2004 by the Josei Rodo Kyokai (the Japan Association for the advancement of working women) reported that $80 \%$ of working women has menstrual pain and 3\% of women had severe symptoms resulting in absence from work. ${ }^{22}$ The prevalence of dysmenorrhea in this JMDC database was only $1.6 \%$, and it is possible that the restriction to patients with at least two recorded diagnoses within 3 months led to the exclusion of less severe cases.

Compared to a study by Tanaka et al, ${ }^{1}$ which was a population-based survey of Japanese women aged 15-49 years, patients in this analysis had a higher rate of LEP utilization. One potential explanation is an increase in the general utilization of LEPs over time that has been captured by our more recently collected JMDC data. Comparatively, our analysis also has a more restrictive patient sample than that of Tanaka et al, ${ }^{2}$ who surveyed women with menstrual symptoms in general, as our study only included patients who consulted for dysmenorrhea at least two times within 3 months, and not for any other menstrual symptoms. In addition to an estimate of prevalence and resource utilization, Tanaka et $\mathrm{al}^{1}$ estimated the "willingness to pay" for a drug that would eliminate all menstrual symptoms. The average willingness-to-pay amount for a drug that would eliminate all menstrual symptoms in outpatients was 4,834 JPY (48 USD) per month. Furthermore, willingness-to-pay to eliminate interference of their menstrual symptoms with the activities of daily life was estimated at 3,304 JPY (33 USD) per month. Interestingly, the annual pharmacy costs attributable to dysmenorrhea, which were derived from differences of annual pharmacy costs between cases and controls in the current analysis, were estimated to be 31,840 JPY (318 USD), or 2,653 JPY (26.5 USD) per month, which was below those of the average monthly costs that outpatients in the aforementioned survey were willing to pay for a drug that would eliminate all menstrual symptoms, and costs that would eliminate interference with daily life due to menstrual symptoms. However, the JMDC pharmacy costs might be less than the pharmacy costs that patients actually paid, as the analysis excluded nonreimbursed therapies, such as NSAIDs and COCs. Therefore, it is uncertain that the total pharmacy costs paid by patients and health insurance are below the willingness-to-pay amount for dysmenorrhea treatments. 
Study limitations include those related to any retrospective claims data analysis, including the lack of data supporting the specific reason for each treatment choice. Furthermore, dysmenorrhea is certainly underreported in claims databases and women included in this study may represent severe cases consulting a practitioner. It was previously estimated that $64.6 \%$ of women with menstrual symptoms do not seek medical care. ${ }^{1}$ In addition, treatment patterns observed in this analysis may not fully reflect all medications taken to treat dysmenorrhea if they are not reimbursed by insurance, such as COCs or over-the-counter pain medications such as NSAIDs. As a result, women who were identified as having 'no treatment' may have received nonreimbursed or over-the-counter therapies during the study period; this could have artificially increased the observed 'no treatment' rate. Because the JMDC is an administrative database, its diagnostic reporting may be incomplete as physicians may report codes for which reimbursement is provided instead of the full or actual diagnoses. For example, NSAIDs prescribed for diagnosis with other pain symptoms not associated with menstruation were not included in this study. Patients who require surgery may not receive a diagnosis of dysmenorrhea as they will instead receive a diagnostic code for the underlying condition requiring surgery (eg, fibroids). The burden of the disease was estimated over the first year of follow-up and not over the whole available period. Building on this research, future analyses may look at costs beyond 12 months, which are likely lower than in the year following diagnosis. Finally, assumptions used to define treatment patterns were somewhat arbitrary, including the use of 90 days as the cut-off point for treatment discontinuation. These assumptions were, however, supported by expert opinion, owing to the fact that physicians typically prescribe such therapies for a maximum of 3 months.

\section{Conclusion}

Considerable heterogeneity in treatment patterns was observed among patients with dysmenorrhea, with relatively low utilization of LEPs in patients with primary dysmenorrhea compared to secondary dysmenorrhea, and those treated by internal medicine physicians compared to obstetricians and gynecologists. Total health care costs were significantly higher among women with dysmenorrhea compared with similar women who do not suffer from this condition, and excess costs are primarily driven by outpatient care. Further research is recommended to evaluate whether a different allocation of resources, for example with higher utilization of LEPs, may yield better health outcomes and reduce the economic burden of dysmenorrhea.

\section{Acknowledgments}

The authors thank Bruno Rossi, formerly with Bayer Yakuhin Ltd., for his contribution to the planning of this research and review of this manuscript. The authors also thank Marcia Reinhart for her medical writing assistance.

\section{Author contributions}

Yutaka Osuga, Erika Tanaka, and Yoshie Onishi designed the study and wrote the protocol. Olivier Cristeau and Yoshie Onishi wrote the statistical analysis plan. Olivier Cristeau performed the statistical analyses. Yutaka Osuga, Erika Tanaka, and Sayako Akiyama interpreted the results. All authors contributed to critically revising the manuscript and have approved the final manuscript.

\section{Disclosure}

This study was funded by Bayer Yakuhin Ltd. Sayako Akiyama and Erika Tanaka are employees of Bayer Yakuhin Ltd. Olivier Cristeau and Yoshie Onishi are employees from Creativ-Ceutical, which received funding from Bayer Yakuhin Ltd. Yutaka Osuga has received funding from Bayer Yakuhin, Ltd. during the conduct of the study. He has received funding outside the study from Bayer Yakuhin, Ltd., Nippon Shinyaku Co., Ltd., Fuji Pharma Co., Ltd., and Mochida Pharmaceutical Co., Ltd. Marcia Reinhart provided writing assistance in the production of this manuscript. The authors report no other conflicts of interest in this work.

\section{References}

1. Tanaka E, Momoeda M, Osuga Y, et al. Burden of menstrual symptoms in Japanese women - an analysis of medical care-seeking behavior from a survey-based study. Int J Womens Health. 2013;6:11-23.

2. Tanaka E, Momoeda M, Osuga Y, et al. Burden of menstrual symptoms in Japanese women: results from a survey-based study. JMed Econ. 2013; 16(11):1255-1266.

3. Yamamoto K, Okazaki A, Sakamoto Y, Funatsu M. The relationship between premenstrual symptoms, menstrual pain, irregular menstrual cycles, and psychosocial stress among Japanese college students. J Physiol Anthropol. 2009;28(3):129-136.

4. Daley AJ. Exercise and primary dysmenorrhoea: a comprehensive and critical review of the literature. Sports Med. 2008;38(8):659-670.

5. Osuga Y, Hayashi K, Kobayashi Y, et al. Dysmenorrhea in Japanese women. Int J Gynaecol Obstet. 2005;88(1):82-83.

6. Iwasa K, Kadowaki J. Clinical condition and diagnosis of dysmenorrhea. Sanka to Fujinka. 2011;78(11):1315-1319.

7. Iacovides S, Avidon I, Bentley A, Baker FC. Reduced quality of life when experiencing menstrual pain in women with primary dysmenorrhea. Acta Obstet Gynecol Scand. 2014;93(2):213-217.

8. Unsal A, Ayranci U, Tozun M, Arslan G, Calik E. Prevalence of dysmenorrhea and its effect on quality of life among a group of female university students. Ups J Med Sci. 2010;115(2):138-145. 
9. Gath D, Osborn M, Bungay G, et al. Psychiatric disorder and gynaecological symptoms in middle aged women: a community survey. $\mathrm{Br}$ Med J (Clin Res Ed). 1987;294(6566):213-218.

10. Takeda T, Wong T, Adachi T, et al. Guidelines for office gynecology in Japan: Japan Society of Obstetrics and Gynecology and Japan Association of Obstetricians and Gynecologists 2011 edition. J Obstet Gynaecol Res. 2012;38(4):615-631.

11. Lefebvre G, Pinsonneault O, Antao V, et al. Primary dysmenorrhea consensus guideline. JObstet Gynaecol Can. 2005;27(12):1117-1146.

12. Osayande AS, Mehulic S. Diagnosis and initial management of dysmenorrhea. Am Fam Physician. 2014;89(5):341-346.

13. ACOG Practice Bulletin No. 110: noncontraceptive uses of hormonal contraceptives. Obstet Gynecol. 2010;115(1):206-218.

14. Cobellis L, Razzi S, Fava A, Severi FM, Igarashi M, Petraglia F. A danazol-loaded intrauterine device decreases dysmenorrhea, pelvic pain, and dyspareunia associated with endometriosis. Fertil Steril. 2004; 82(1):239-240.

15. Magon N. Gonadotropin releasing hormone agonists: Expanding vistas. Indian J Endocrinol Metab. 2011;15(4):261-267.

16. Henzl MR, Corson SL, Moghissi K, Buttram VC, Berqvist C, Jacobson $\mathrm{J}$. Administration of nasal nafarelin as compared with oral danazol for endometriosis. A multicenter double-blind comparative clinical trial. N Engl J Med. 1988;318(8):485-489.
17. Henzl MR, Kwei L. Efficacy and safety of nafarelin in the treatment of endometriosis. Am J Obstet Gynecol. 1990;162(2):570-574.

18. Medical Information System Development Center. Master of diseases and Injuries Names. In: MEDIS-DC. Tokyo, Japan: Medical Information System Development Center; 2014.

19. Copher R, Le NE, Law A, Pocoski J, Zampaglione E. Retrospective analysis of variation in heavy menstrual bleeding treatments by age and underlying cause. Curr Med Res Opin. 2013;29(2): 127-139.

20. Copher R, Le NE, Zampaglione E, Prezioso A, Pocoski J, Law A. Heavy menstrual bleeding treatment patterns and associated health care utilization and costs. J Clin Outcom Manag. 2012;19:402-413.

21. Ohde S, Tokuda Y, Takahashi O, Yanai H, Hinohara S, Fukui T. Dysmenorrhea among Japanese women. Int J Gynaecol Obstet. 2008;100(1): 13-17.

22. Josei Rodo Kyokai (Japan Association for the Advancement of Working Women). Survey report on the health of working women. 2014. Available from: http://www.jaaww.or.jp/index.php. Accessed November 15, 2016.

23. Health Insurance Bureau, Ministry of Health, Labor and Welfare. The medical procedure index. Available from: http://www.iryohoken.go.jp/ shinryohoshu/. Accessed May 15, 2017.

\section{ClinicoEconomics and Outcomes Research}

\section{Publish your work in this journal}

ClinicoEconomics and Outcomes Research is an international, peerreviewed open-access journal focusing on health technology assessment, pharmacoeconomics and outcomes research in the areas of diagnosis, medical devices, and clinical, surgical and pharmacological intervention. The economic impact of health policy and health systems
Submit your manuscript here: https://www.dovepress.com/clinicoeconomics-and-outcomes-research-jourat

organization also constitute important areas of coverage. The manuscript management system is completely online and includes a very quick and fair peer-review system, which is all easy to use. Visit http://www.dovepress.com/testimonials.php to read real quotes from published authors. 